\title{
Viscoelastic fishbones
}

\author{
Bavand Keshavarz ${ }^{\oplus},{ }^{*}$ Michela Geri, and Gareth H. McKinley \\ Hatsopoulos Microfluids Laboratory, Department of Mechanical Engineering, \\ Massachusetts Institute of Technology, Cambridge, Massachusetts 02139, USA
}

(Received 22 March 2019; published 24 October 2019)

\begin{abstract}
This paper is associated with a poster winner of a 2018 APS/DFD Milton van Dyke Award for work presented at the DFD Gallery of Fluid Motion. The original poster is available from the Gallery of Fluid Motion, https://doi.org/10.1103/APS.DFD.2018.GFM. P0045.
\end{abstract}

DOI: 10.1103/PhysRevFluids.4.100509

Two identical fast liquid jets collide into each other at an oblique angle, as seen in Fig. 1(a). To the naked eye they form a blurred liquid fan, as first observed by the German scientist H. G. Magnus [1,2] and later studied in detail by Bush and Hasha [3] along with Villermaux and coworkers [4,5]. Inspired by the still images of flying bullets from H. E. "Doc" Edgerton (MIT pioneer of high-speed imaging) [6], we visualize these phenomena using a high-speed air-gap flash that releases a 20000 -volt arc into air in less than $300 \mathrm{~ns}$. Liquid patterns are then frozen in time.

As shown in Fig. 1(b), the Newtonian liquid sheet breaks into a chain of droplets that depart from the liquid "fishbone." Each droplet (filament) formed acts as a spherical (cylindrical) "liquid lens." As shown in Fig. 1(c), using the lens effect, inside each droplet we can see a focused image of the unfocused background. The falling "APS peaches" of the Newtonian liquid form a group of droplets with a remarkably monodisperse size distribution [Fig. 1(b)].

For viscoelastic liquids, the nonlinear dynamics of elongating macromolecules can delay the capillary breakup event and inhibit the fragmentation process [7-10]. In order to demonstrate this, we prepare a dilute polymeric solution by dissolving poly(ethylene oxide) with molecular weight $M_{w}=1000 \mathrm{~kg} / \mathrm{mol}$, in the Newtonian solvent (1-1 water-glycerol mixture) at a weight concentration of $c=0.01 \%$ corresponding to a value of $c / c^{*}=0.07$ where $c^{*}$ is the overlap concentration [9-11]. As shown in Fig. 2, for the viscoelastic fluid, the enhanced elongational viscosity delays the breakup of the liquid filaments that emerge from the sheet. This leads to the formation of beads and elongated filaments with corrugated geometries. The emergence of

\footnotetext{
*bavand@mit.edu
}

Published by the American Physical Society under the terms of the Creative Commons Attribution 4.0 International license. Further distribution of this work must maintain attribution to the author(s) and the published article's title, journal citation, and DOI. 

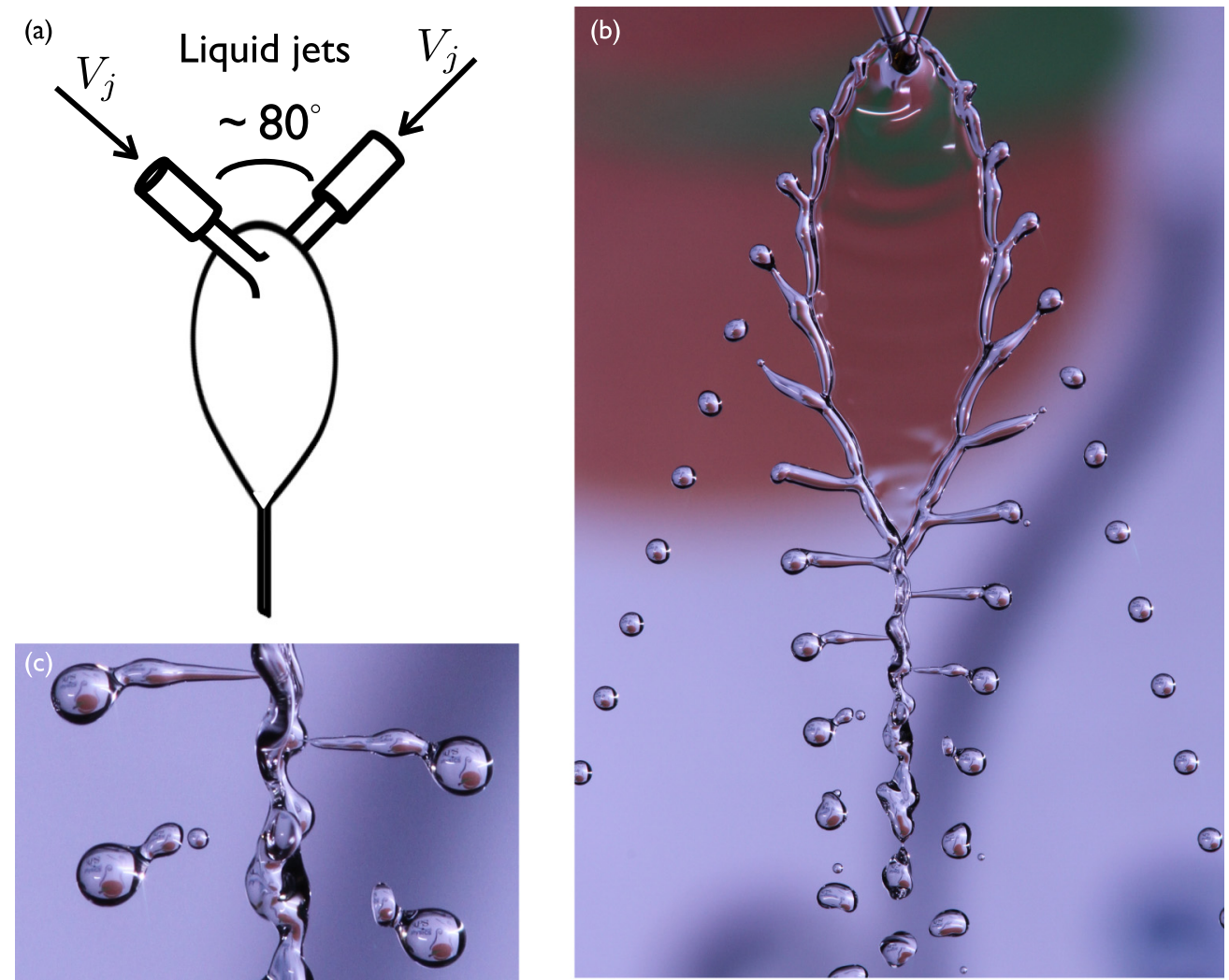

FIG. 1. (a) Our experimental setup: two identical Newtonian jets (water and glycerol mixed at 1:1 volume ratio), exit from two $0.61 \mathrm{~mm}$ diameter cylindrical nozzles. (b) The Newtonian jets collide and a liquid sheet is formed. Each jet has a radius of $R_{j}=0.3 \mathrm{~mm}$ and a velocity of $V_{j}=2.85 \mathrm{~m} / \mathrm{s}$. The relevant dimensionless numbers in this experiment are as follows: Weber number $\mathrm{We}_{j} \equiv \rho V_{j}^{2} R / \sigma=392$, Ohnesorge number $\mathrm{Oh}_{j}=$ $\mu / \sqrt{\rho \sigma R_{j}}=0.043$, and Deborah number $\operatorname{De}_{j}=\tau_{E} / \sqrt{\rho R_{j}^{3} / \sigma}=0$ where $\rho, \sigma, \mu$, and $\tau_{E}$ are respectively the density, surface tension, viscosity, and elongational relaxation time of the fluid. (c) A magnified view shows a focused image of the background picture inside each droplet or filament (see the text for further details). See https://doi.org/10.1103/APS.DFD.2018.GFM.P0045.

corrugated ligaments in the fragmentation of viscoelastic liquids makes the final droplet size distributions markedly broader (compared to the corresponding distributions for the atomization of the Newtonian solvent) [10].

Using the same lens principle for spherical (cylindrical) droplets (filaments), we can introduce an optical flow tracer. As shown in Fig. 2, for the viscoelastic case the Society of Rheology's hourglass logo is positioned in the background in such a way that "the sands of time are stretched in the filaments and then relax in the droplets"; an indirect reference to the fact that polymer chains stretch in the filaments and relax in the droplets. 

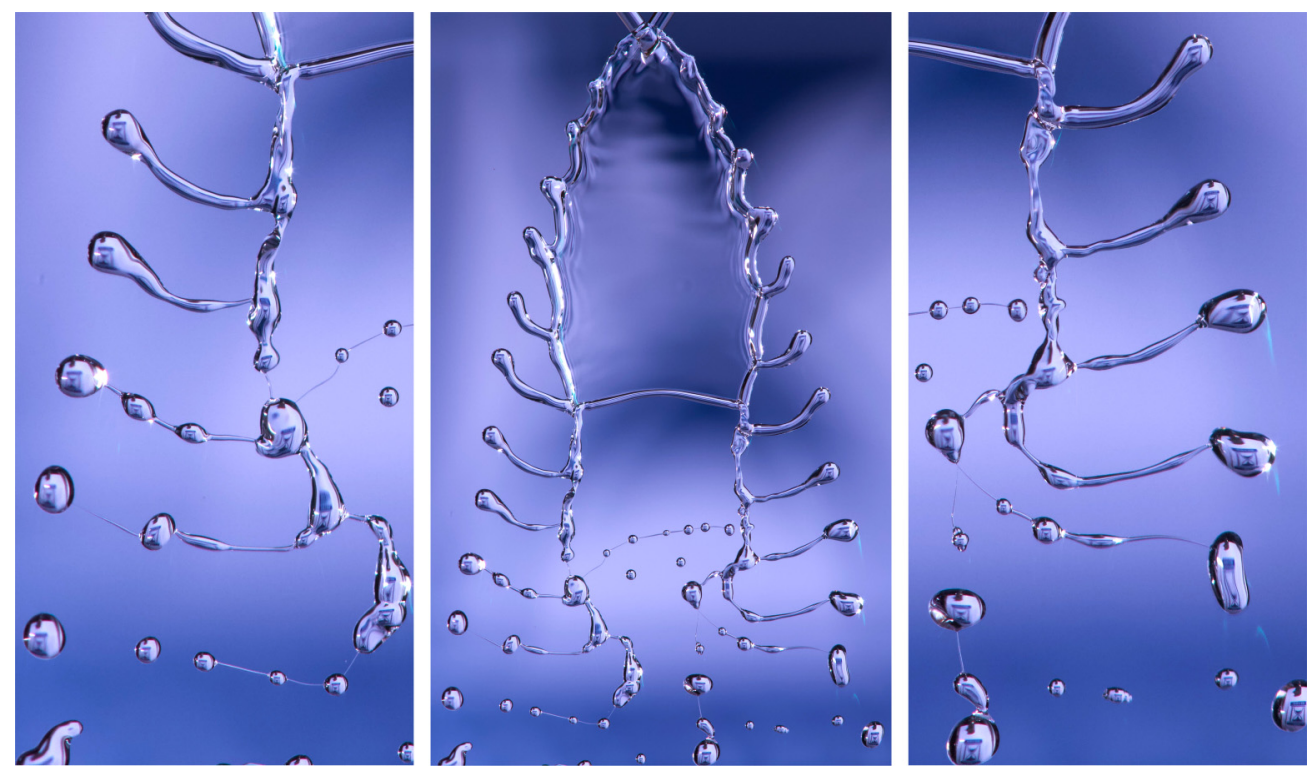

FIG. 2. Center: Two identical viscoelastic jets collide and a liquid sheet is formed. Each jet has a radius of $R_{j}=0.3 \mathrm{~mm}$ and a velocity of $V_{j}=2.85 \mathrm{~m} / \mathrm{s}\left(\mathrm{We}_{j} \equiv \rho V_{j}^{2} R / \sigma=392, \mathrm{Oh}_{j}=\mu / \sqrt{\rho \sigma R_{j}}=0.043\right.$, and $\mathrm{De}_{j}=$ $\tau_{E} / \sqrt{\rho R_{j}^{3} / \sigma}=1.49$ ). The right-hand and left-hand panels show a magnified view of the stretched ligaments and the beads-on-a-string structures, respectively. See https://doi.org/10.1103/APS.DFD.2018.GFM.P0045.

[1] G. Magnus, Hydraulische untersuchungen, Ann. Phys. 171, 1 (1855).

[2] C. Clanet, Waterbells and liquid sheets, Annu. Rev. Fluid Mech. 39, 469 (2006).

[3] J. W. M. Bush and A. E. Hasha, On the collision of laminar jets: Fluid chains and fishbones, J. Fluid Mech. 511, 285 (2004).

[4] E. Villermaux, Fragmentation, Annu. Rev. Fluid Mech. 39, 419 (2007).

[5] N. Bremond and E. Villermaux, Atomization by jet impact, J. Fluid Mech. 549, 273 (2006).

[6] G. Kayafas and E. Jussim, Stopping Time: The Photographs of Harold Edgerton (Harry N. Abrams, 2000).

[7] E. Miller, B. Gibson, E. McWilliams, and J. P. Rothstein, Collision of viscoelastic jets and the formation of fluid webs, Appl. Phys. Lett. 87, 014101 (2005).

[8] S. Jung, S. D. Hoath, G. D. Martin, and I. M. Hutchings, Experimental study of atomization patterns produced by the oblique collision of two viscoelastic liquid jets, J. Non-Newtonian Fluid Mech. 166, 297 (2011).

[9] B. Keshavarz, V. Sharma, E. C. Houze, M. R. Koerner, J. R. Moore, P. M. Cotts, P. Threlfall-Holmes, and G. H. McKinley, Studying the effects of elongational properties on atomization of weakly viscoelastic solutions using Rayleigh Ohnesorge jetting extensional rheometry (ROJER), J. Non-Newtonian Fluid Mech. 222, 171 (2015).

[10] B. Keshavarz, E. C. Houze, J. R. Moore, M. R. Koerner, and G. H. McKinley, Ligament Mediated Fragmentation of Viscoelastic Liquids, Phys. Rev. Lett. 117, 154502 (2016).

[11] M. Rubinstein and R. H. Colby, Polymer Physics (Oxford University Press, Oxford, 2003). 